\title{
2-Dimensional Deformation of an Irregular Orthotropic Elastic Medium
}

\author{
Dinesh Kumar Madan and Aanchal Gaba \\ Department of Mathematics, The Technological Institute of Textile \& Sciences, Bhiwani-127021 (INDIA)
}

\begin{abstract}
In the present paper the closed form analytical expressions for the displacement and stresses at any point of an infinite irregular orthotropic elastic medium as a result of normal line-load have been obtained by using eigen-value approach. The irregularity of rectangular and parabolic shape has been considered. To examine the effect of different type of irregularities the variation of displacement and stresses with horizontal distance have been shown graphically by taking different sizes of irregularities. Also to study the effect of irregularities present in the medium the comparison between the displacement due to with and without irregularities have been made. Contour maps showing the displacement field for each type of irregularities are presented.
\end{abstract}

Keywords: Orthotropic medium, Normal line-load, Irregularity, Rectangular, Parabolic, Eigen value

\section{Introduction}

Although isotropy is a good approximation in the Earth, it is sometimes important to consider departure from isotropy, i.e., anisotropy. From the study on earthquakes and earth structures, it has been observed that the Earth is anisotropic in nature. Most anisotropic medium of interest in seismology have at least approximately a horizontal plane of symmetry. Medium having three orthogonal planes of symmetry is called orthotropic medium. Since the orientation of stern in the Earth's crust is usually orthotropic, 'most symmetry systems in the crust of Earth have orthotropic orientation'. The most important anisotropic materials are Olivine and Orthoyroxenes, which comprise much of the deep crust and upper mental, exhibit orthotropic symmetry. Moreover the interfaces separating the different media of the Earth are not perfectly plane. To better understand the seismic behavior at continental margins, mountain rocks etc., the static deformation problem of an anisotropic elastic medium with irregularities present is very important.

The problems of propagation of seismic waves with irregularities present in the elastic medium have been studied by many researchers like De Noyer [1], A.K. Mal [2] ,Kar et al. [3], Chattopadhyay [4], Acharya and Roy[5], and others. Madan et al. [6] and Kumar et al. [7] analyzed the effect of rigidity and irregularity on the propagation of Love waves in fluid saturated porous anisotropic single layered and multilayered elastic media. Garg et al. [8] studied the plane strain problem of infinite orthotropic elastic medium due to twodimensional sources by considering distinct Eigen values. By using Eigen value method Singh et al. [9] obtained the deformation field for the monoclinic medium in the transformed domain with plane interface. Salim [10] studied the effect of rectangular irregularity on the static deformation of initially stressed and unstressed isotropic elastic medium respectively. In isotropic medium the Eigen values cannot be distinct.

In the present paper we have considered the homogenous, orthotropic elastic medium to study the effect of rectangular and parabolic irregularities on the static deformation due to normal line load. Anisotropy resulted from a material being non-uniform or homogenous. The crystal structure of the mineral Olivine is homogeneous as it is composed of the same repeating groups of atoms, but acts anisotropic because its elastic properties vary in different directions relative to the crystal lattice. Numerically by considering the material Olivine, the effect of irregularities has been examined by drawing the variations of displacements and normal stresses with horizontal distance for different size of irregularities. Also the comparison of displacements for regular medium with rectangular and parabolic irregularities present in the medium has been made graphically. The variations of displacements with different sizes of two different irregularities i.e. rectangular and parabolic are depicted by drawing the contour.

The present problem is an improvement of the earlier papers studied by Garg et al. [8] for regular orthotropic elastic medium and Salim [10] for irregular (rectangular) isotropic medium. The corresponding results for normal loading obtained by Garg et al [8] can be recovered from our results as a particular case.

\section{Formulation of the Problem}

We consider an infinite orthotropic elastic medium with rectangular $\mathrm{x}$ - axis vertically downwards and let the origin of the co-ordinate $(\mathrm{x}, \mathrm{y}, \mathrm{z})$ be situated at $\mathrm{x}=0$. Suppose that a normal line load $F_{0}$, per unit, length, is acting vertically downward on a line parallel to the $\mathrm{z}$ - axis and passing through the points $(\mathrm{H}, 0)$. Let the 
rectangular or parabolic irregularity be present in the medium. The geometry of the problem is shown in figures 1(a) and 1(b).The equation of rectangular and parabolic irregularities may respectively be presented as [5]:

(i) Rectangular irregularity:

$$
x=E f(y)=\left\{\begin{array}{c}
h \text { for }|y| \leq a \\
0 \text { for }|y| \geq a
\end{array}\right.
$$

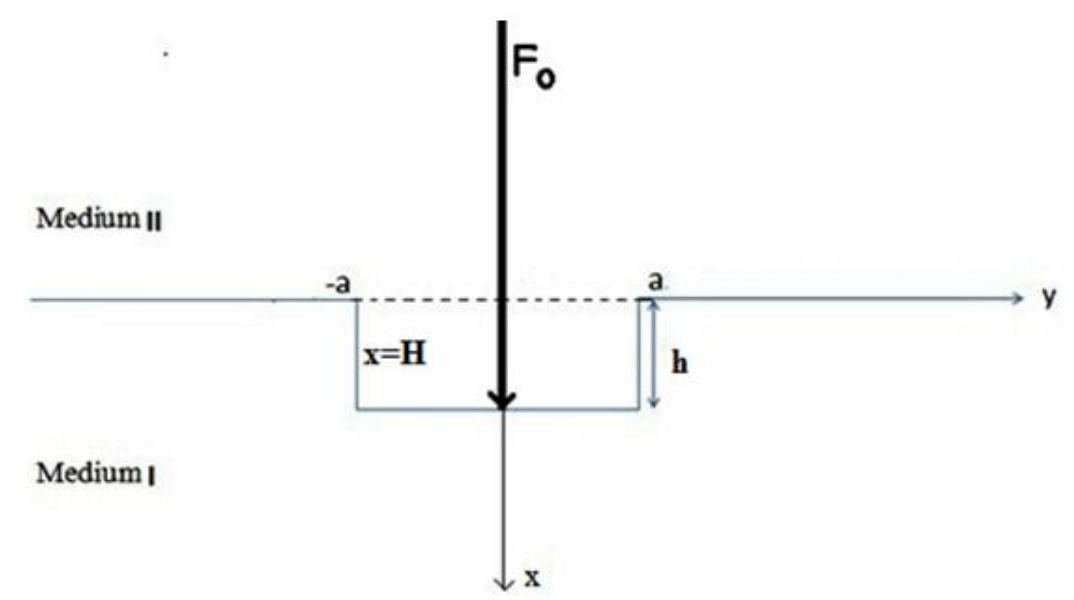

Figure 1(a)

and

(ii) Parabolic

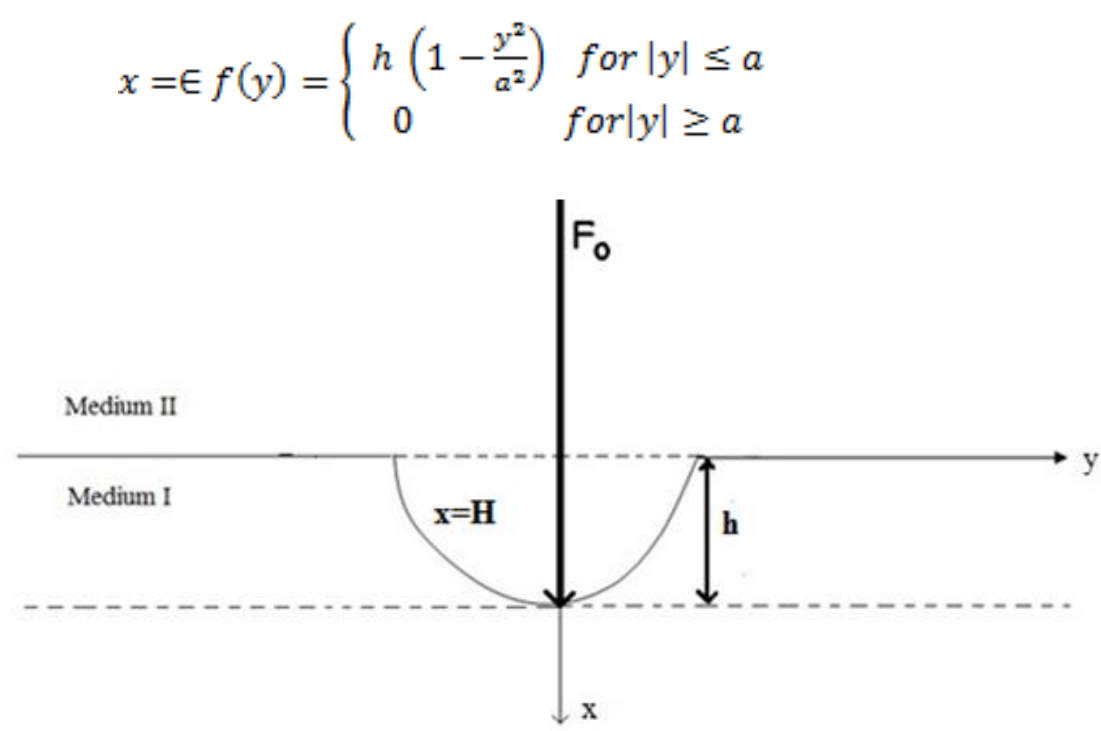

irregularity

Figure 1(b)

where $E=\frac{h}{2 a} \ll 1$ is the perturbation parameter.

\section{Theory}

The equilibrium equations is the Cartesian co-ordinate system $(x, y, z)$ for zero body forces are

$$
\begin{aligned}
& \sigma_{x x}+\tau_{x y y}+\tau_{x z z}=0 \\
& \tau_{y x x}+\sigma_{y y}+\tau_{y z z}=0
\end{aligned}
$$

$\tau_{z x x}+\tau_{z y y}+\sigma_{z z}=0$

where $\sigma_{x,}, \sigma_{y y}, \sigma_{z}$ referred as normal stresses and $\tau_{x y y} \tau_{x z}, \tau_{y x}, \tau_{y z}, \tau_{z x}$ and $\tau_{z y}$ are called shearing stresses.

The stress-strain relations for an orthotropic elastic medium, with co-ordinate planes as planes of elastic symmetry are [11] 
$\sigma_{x}=c_{11} e_{x}+c_{12} e_{y}+c_{13} e_{z}$

$\sigma_{y}=c_{21} e_{x}+c_{22} e_{y}+c_{23} e_{z}$

$\sigma_{z}=c_{13} e_{x}+c_{23} e_{y}+c_{33} e_{z}$

$\tau_{y z}=2 c_{44} e_{y z}$

$\tau_{x z}=2 c_{55} e_{x z}$

$\tau_{x y}=2 c_{66} e_{x y}$

where $e_{x x}, e_{y y}, e_{x}$ are normal strain components and $e_{x y y} e_{y z}, e_{x z}$ are called the shearing strain components. The two suffix quantities $c_{i j}(\mathrm{i}, \mathrm{j}=1,2, \ldots \ldots \ldots, 6)$ are the elastic constants which describe the properties of an orthotropic elastic material. The strain-displacement relations are given as:

$e_{x y}=\frac{1}{2}\left(\frac{\partial u}{\partial y}+\frac{\partial v}{\partial x}\right) \quad$ and $e_{x}=\frac{\partial u}{\partial x}$ etc.

The equilibrium equation in terms of displacements components can be obtained from equations (3)-(7). We find

$$
\begin{aligned}
& c_{11} \frac{\partial^{2} u}{\partial x^{2}}+c_{66} \frac{\partial^{2} u}{\partial y^{2}}+c_{55} \frac{\partial^{2} u}{\partial z^{2}}+\left(c_{12}+c_{66}\right) \frac{\partial^{2} w}{\partial x \partial y}+\left(c_{13}+c_{55}\right) \frac{\partial^{2} w}{\partial x \partial z}=0 \\
& \left(c_{66}+c_{12}\right) \frac{\partial^{2} u}{\partial x \partial y}+c_{66} \frac{\partial^{2} v}{\partial x^{2}}+c_{22} \frac{\partial^{2} v}{\partial y^{2}}+c_{44} \frac{\partial^{2} v}{\partial z^{2}}+\left(c_{23}+c_{44}\right) \frac{\partial^{2} w}{\partial z \partial y}=0 \\
& \left(c_{55}+c_{13}\right) \frac{\partial^{2} u}{\partial x \partial z}+\left(c_{44}+c_{23}\right) \frac{\partial^{2} v}{\partial z \partial y}+c_{55} \frac{\partial^{2} w}{\partial x^{2}}+c_{44} \frac{\partial^{2} w}{\partial y^{2}}+c_{33} \frac{\partial^{2} w}{\partial z^{2}}=0
\end{aligned}
$$

We consider plane strain deformation, parallel to $x y-p l a n e$, in which the displacement components are independent of $\mathrm{z}$ and are of the type

$u=u(x, y), v=v(x, y), w=0$

The non-zero stresses for the plane strain problem are

$$
\begin{aligned}
& \sigma_{x}=c_{11} \frac{\partial u}{\partial x}+c_{12} \frac{\partial v}{\partial y} \\
& \sigma_{y}=c_{12} \frac{\partial u}{\partial x}+c_{22} \frac{\partial v}{\partial y} \\
& \sigma_{z}=c_{13} \frac{\partial u}{\partial x}+c_{23} \frac{\partial v}{\partial y} \\
& \tau_{x y}=c_{66}\left(\frac{\partial u}{\partial x}+\frac{\partial v}{\partial y}\right)
\end{aligned}
$$

The equilibrium equations for an orthotropic elastic medium due to plane strain deformation are to be found from equations (8)-(11), we find

$$
\begin{aligned}
& c_{11} \frac{\partial^{2} u}{\partial x^{2}}+c_{66} \frac{\partial^{2} u}{\partial y^{2}}+\left(c_{66}+c_{12}\right) \frac{\partial^{2} v}{\partial x \partial y}=0 \\
& \left(c_{66}+c_{12}\right) \frac{\partial^{2} u}{\partial x \partial y}+c_{66} \frac{\partial^{2} v}{\partial x^{2}}+c_{22} \frac{\partial^{2} v}{\partial y^{2}}=0
\end{aligned}
$$

and equilibrium equation (10) becomes an identity.

We define Fourier transform $\bar{f}(\mathrm{k})$ of $\mathrm{f}(\mathrm{y})$ by the relation [12]

$\bar{f}(\mathrm{k})=\mathrm{F}[\mathrm{f}(\mathrm{y})]=\int_{-\infty}^{\infty} f(y) e^{i k y} d y$

So that

$\mathrm{f}(\mathrm{y})=\frac{1}{2 \pi} \int_{-\infty}^{\infty} \bar{f}(k) e^{-i k y} d k$

where $\mathrm{k}$ is the transform Fourier parameter.

Applying the Fourier transformation on equilibrium equations (16) and (17) for an orthotropic elastic medium, we obtain

$$
\begin{aligned}
& c_{11} \frac{d^{2} \bar{u}}{d x^{2}}-k^{2} c_{66} \bar{u}+\left(c_{66}+c_{12}\right)(-i k) \frac{d \bar{v}}{d x}=0 \\
& \left(c_{66}+c_{12}\right)(-i k) \frac{d \bar{u}}{d x}+c_{66} \frac{d^{2} \bar{u}}{d x^{2}}+c_{22}\left(-k^{2} \bar{v}\right)=0
\end{aligned}
$$

In vector-matrix differential equation form, equations (20) and (21) can be written as:

$\mathrm{A} \frac{d^{2} U}{d x^{2}}-i k B \frac{d U}{d x}-k^{2} C U=0$ 
where

$A=\left(\begin{array}{cc}c_{11} & 0 \\ 0 & c_{66}\end{array}\right)$
$B=\left(\begin{array}{cc}0 & c_{66}+c_{12} \\ c_{66}+c_{12} & 0\end{array}\right)$
$\mathrm{C}=\left(\begin{array}{cc}c_{66} & 0 \\ 0 & c_{22}\end{array}\right)$
$U=\left(\begin{array}{l}\bar{u} \\ \bar{v}\end{array}\right)$

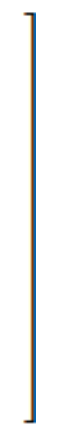

We note that matrices A, B, C are all symmetric. Now we by a solution of the matrix equation (22) of the form

$U(x, k)=E(k) e^{m x}$

(24)

Where $\mathrm{m}$ is a parameter and $\mathrm{E}(\bar{k})$ is a matrix of the type $2 \times 1$.

From equations (24)-(26), the characteristics equation is obtained as:

$\left(c_{11} c_{66}\right) m^{4}-\left(c_{11} c_{12}-2 c_{66} c_{12}-c^{2}{ }_{12}\right) k^{2} m^{2}+\left(c_{66} c_{22}\right) k^{4}=0$

(25)

when the medium is transversely isotropic [13]:

$c_{22}=c_{11}$

$c_{23}=c_{13}$

$c_{55}=c_{44}$

$c_{66}=1 / 2\left(c_{11}-c_{12}\right)$

and when the medium is isotropic:

$\mathrm{c}_{11}=\mathrm{c}_{22}=\mathrm{c}_{33}=\lambda+2 \mu, \mathrm{c}_{12}=\mathrm{c}_{13}=\mathrm{c}_{23}=\lambda, \mathrm{c}_{44}=\mathrm{c}_{55}=\mathrm{c}_{66}=\mu$

Where $\lambda$ and $\mu$ are the Lame elastic moduli. The characteristics equation (25) for both transversely and isotropic elastic medium becomes

$m^{4}-2 k^{2} m^{2}+k^{4}=0$

We observe that the characteristics equation (28) is independent of its elastic moduli as compared to orthotropic elastic medium.

The roots of the characteristic equation (25) gives the eigen values $\mathrm{m}=$ corresponding eigen-vectors are obtained by solving the equation:

$\left[m^{2}-i m k B-k^{2} C\right] E(k)=0$

$m_{1}, m_{2}, m_{3}$ and $m_{4}$ and

On solving the equation (25) and correspondingly equation, we have

$m_{1}=\alpha_{1}|k|, m_{2}=\alpha_{2}|k|, m_{3}=-\alpha_{1}|k|, m_{4}=-\alpha_{2}|k|$

where

$\alpha_{1}^{2}=\frac{A_{0}+\sqrt{A_{0}{ }^{2}-4 B_{0}}}{2}, \alpha_{2}{ }^{2}=\frac{A_{0}-\sqrt{A_{0}{ }^{2}-4 B_{0}}}{2}$

$A_{0}=\frac{c_{11} c_{22}-2 c_{66} c_{12}-c_{12}^{2}}{c_{11} c_{66}}, B_{0}=c_{22} / c_{11}$.

We note that $\alpha_{1}$ and $\alpha_{2}$ for an orthotropic elastic medium depends upon elastic moduli only (and not on $\mathrm{k})$ and they may be real or complex. We assume that $\left(\alpha_{1} \neq \alpha_{2}\right)$ for an orthotropic medium.

And the eigen-vectors for an orthotropic elastic medium are found to be

$E_{N}{ }^{T}=\left[P_{N}, 1\right], \quad E_{N+2}{ }^{T}=\left[-P_{N}, 1\right]$

Where

$P_{N}=i \frac{\alpha_{N}\left(c_{66}+c_{12}\right)}{\alpha_{N}{ }^{2} c_{11}-c_{66}}=-i \frac{\alpha_{n}{ }^{2} c_{66}-c_{22}}{\alpha_{N}\left(c_{66}+c_{12}\right)} \quad$ for $\mathrm{N}=1,2$

Therefore, a solution of square matrix (22) for the case of an orthotropic elastic medium is

$U(x, k)=\left(B E_{1}{ }^{T} e^{\alpha_{1}|k| x}+D E_{2}{ }^{T} e^{\alpha_{2}|k| x}\right)+\left(C E_{1}{ }^{T} e^{\alpha_{1}|k| x}+G E_{2}{ }^{T} e^{\alpha_{2}|k| x}\right)$

Where B, C, D, G are constants to be determined from boundary conditions and they may depend upon the parameter $\mathrm{k}$.From equations (23), (33) and (35), we write

$\bar{u}(x, y)=B P_{1} e^{\alpha_{1}|k| x}+C P_{2} e^{\alpha_{2}|k| x}-D P_{1} e^{-\alpha_{1}|k| x}-G P_{2} e^{-\alpha_{2}|k| x}$ 
$\bar{v}(x, y)=B P_{1} e^{\alpha_{1}|k| x}+C P_{2} e^{\alpha_{2}|k| x}+D P_{1} e^{-\alpha_{1}|k| x}+G P_{2} e^{-\alpha_{2}|k| x}$

Inversion of equations (36) and (37) yield the displacements in the following integral forms

$\mathrm{u}(\mathrm{x}, \mathrm{y})=\frac{1}{2 \pi} \int_{-\infty}^{\infty}\left(B P_{1} e^{\alpha_{1}|k| x}+C P_{2} e^{\alpha_{2}|k| x}-D P_{1} e^{-\alpha_{1}|k| x}-G P_{2} e^{-\alpha_{2}|k| x}\right) e^{-i k y} d k$

$\mathrm{v}(\mathrm{x}, \mathrm{y})=\frac{1}{2 \pi} \int_{-\infty}^{\infty}\left(B P_{1} e^{\alpha_{1}|k| x}+C P_{2} e^{\alpha_{2}|k| x}+D P_{1} e^{-\alpha_{1}|k| x}+G P_{2} e^{-\alpha_{2}|k| x}\right) e^{-i k y} d k$

The stress components in integral forms for an orthotropic elastic medium due to plane strain deformation are obtained from equations (16), (38) and (39).

$\sigma_{x}=\frac{1}{2 \pi} \int_{-\infty}^{\infty}\left(Q_{1} B e^{\alpha_{1}|k| x}+Q_{2} C e^{\alpha_{2}|k| x}+Q_{1} D e^{-\alpha_{1}|k| x}+Q_{2} G e^{-\alpha_{2}|k| x}\right) e^{-i k y} d k$

$\tau_{x y}=\frac{1}{2 \pi} \int_{-\infty}^{\infty} c_{66}\left(R_{1} B e^{\alpha_{1}|k| x}+R_{2} C e^{\alpha_{2}|k| x}+R_{1} D e^{-\alpha_{1}|k| x}+R_{2} G e^{-\alpha_{2}|k| x}\right) e^{-i k y} d k$

$\mathrm{Q}_{N}=c_{11} \alpha_{N}|k|-i c_{12} k \quad, R_{N}=\alpha_{N}|\mathrm{k}|-\mathrm{i} P_{N} k$

\section{Solution of the Problem}

To find the elastic field at any point of an irregular orthotropic elastic infinite medium due to a normal line-load, we consider the infinite medium consisting of Medium I $(x>0)$ and Medium II $(x<0)$ of identical elastic properties.

For Medium I, the displacement and stress components are:

$$
\begin{aligned}
& \mathrm{u}^{\mathrm{I}}(\mathrm{x}, \mathrm{y})=\frac{1}{2 \pi} \int_{-\infty}^{\infty}\left(-D P_{1} e^{-\alpha_{1}|k| x}-G P_{2} e^{-\alpha_{2}|k| x}\right) e^{-i k y} d k \\
& v^{I}(\mathrm{x}, \mathrm{y})=\frac{1}{2 \pi} \int_{-\infty}^{\infty}\left(D e^{-\alpha_{1}|k| x}+G e^{-\alpha_{2}|k| x}\right) e^{-i k y} d k \\
& \left.\sigma_{x}^{I}(x, y)=\frac{1}{2 \pi} \int_{-\infty}^{\infty} Q_{1} D e^{-\alpha_{1}|k| x}+Q_{2} G e^{-\alpha_{2}|k| x}\right) e^{-i k y} d k \\
& \tau_{x y}{ }^{I}(x, y)=\frac{1}{2 \pi} \int_{-\infty}^{\infty} c_{66}\left(-R_{1} D e^{-\alpha_{1}|k| x}-R_{2} G e^{-\alpha_{2}|k| x}\right) e^{-i k y} d k
\end{aligned}
$$

And the displacement and stresses components for the medium II are

$\mathrm{u}^{\mathrm{II}}(\mathrm{x}, \mathrm{y})=\frac{1}{2 \pi} \int_{-\infty}^{\infty}\left(B P_{1} e^{\alpha_{1}|k| x}+C P_{2} e^{\alpha_{2}|k| x}\right) e^{-i k y} d k$

$v^{I I}(\mathrm{x}, \mathrm{y})=\frac{1}{2 \pi} \int_{-\infty}^{\infty}\left(B e^{\alpha_{1}|k| x}+C e^{\alpha_{2}|k| x}\right) e^{-i k y} d k$

$\left.\sigma_{x}^{I I}(x, y)=\frac{1}{2 \pi} \int_{-\infty}^{\infty} Q_{1} B e^{\alpha_{1}|k| x}+Q_{2} C e^{\alpha_{2}|k| x}\right) e^{-i k y} d k$

$\tau_{x y}{ }^{I I}(x, y)=\frac{1}{2 \pi} \int_{-\infty}^{\infty} c_{66}\left(R_{1} B e^{\alpha_{1}|k| x}+R_{2} C e^{\alpha_{2}|k| x}\right) e^{-i k y} d k$

The boundary conditions are:

$$
\begin{aligned}
& u^{2}(H, y)=u^{I I}(H, y) \\
& v^{2}(H, y)=v^{I I}(H, y) \\
& \sigma_{x}{ }^{2}(H, y)-\sigma_{x}{ }^{I I}(H, y)=-F_{0} \delta(y) \\
& \tau_{x y}{ }^{2}(H, y)=\tau_{x y}{ }^{I I}(H, y)
\end{aligned}
$$

Where $H=\in f(y)$ and $\delta(y)$ is the Dirac delta function satisfying the following property

$\int_{-\infty}^{\infty} \delta(y) d y=1, \delta(y)=\frac{1}{2 \pi} \int_{-\infty}^{\infty} e^{-i k y} d k$

Following the method of Eringen and Suhuni [14] the arbitrary function B, C, D, and G can be evaluated by expanding these in terms of $\in$ and retaining only the linear terms, we may write

$\mathrm{B}=B_{0}+\epsilon B_{1}, \mathrm{C}=C_{0}+\epsilon C_{1}, \mathrm{D}=D_{0}+\epsilon D_{1}, \mathrm{G}=G_{0}+\epsilon G_{1}$

At $\mathrm{x}=H$ and using equation (52), equations (43)-(50) can be written as:

$\mathrm{u}^{\mathrm{I}}(H, \mathrm{y})=\frac{1}{2 \pi} \int_{-\infty}^{\infty}\left(-\left(D_{0}+\epsilon D_{1}\right) P_{1} e^{-\alpha_{1}|k| \Delta}-\left(G_{0}+\epsilon G_{1}\right) P_{2} e^{-\alpha_{2}|k| \Delta}\right) e^{-i k y} d k$

$v^{I}(H, \mathrm{y})=\frac{1}{2 \pi} \int_{-\infty}^{\infty}\left(\left(D_{0}+\epsilon D_{1}\right) e^{-\alpha_{1}|k| \Delta}+\left(G_{0}+\epsilon G_{1}\right) e^{-\alpha_{2}|k| \Delta}\right) e^{-i k y} d k$

$\left.\sigma_{x}{ }^{I}(H, y)=\frac{1}{2 \pi} \int_{-\infty}^{\infty} Q_{1}\left(D_{0}+\epsilon D_{1}\right) e^{-\alpha_{1}|k| \Delta}+Q_{2}\left(G_{0}+\epsilon G_{1}\right) e^{-\alpha_{2}|k| \Delta}\right) e^{-i k y} d k$

$\tau_{x y}^{I}\left(H_{y} y\right)=\frac{1}{2 \pi} \int_{-\infty}^{\infty} c_{66}\left(-R_{1}\left(D_{0}+\epsilon D_{1}\right) e^{-\alpha_{1}|k| \Delta}-R_{2}\left(G_{0}+\epsilon G_{1}\right) e^{-\alpha_{2}|k| x}\right) e^{-i k \Delta} d k$

And for medium $\square \square$

$\mathrm{u}^{\mathrm{II}}(H, \mathrm{y})=\frac{1}{2 \pi} \int_{-\infty}^{\infty}\left(\left(B_{0}+\epsilon B_{1}\right) P_{1} e^{\alpha_{1}|k| \Delta}+\left(C_{0}+\epsilon C_{1}\right) P_{2} e^{\alpha_{2}|k| \Delta}\right) e^{-i k y} d k$

$v^{I I}(H, \mathrm{y})=\frac{1}{2 \pi} \int_{-\infty}^{\infty}\left(\left(B_{0}+\epsilon B_{1}\right) e^{\alpha_{1}|k| \Delta}+\left(C_{0}+\epsilon C_{1}\right) e^{\alpha_{2}|k| \Delta}\right) e^{-i k y} d k$ 
$\sigma_{x}^{I I}(H, y)=\frac{1}{2 \pi} \int_{-\infty}^{\infty}\left(Q_{1}\left(B_{0}+\epsilon B_{1}\right) e^{\alpha_{1}|k| \Delta}+Q_{2}\left(C_{0}+\epsilon C_{1}\right) e^{\alpha_{2}|k| \Delta}\right) e^{-i k y} d k$

$\tau_{x y}{ }^{I I}(H, y)=\frac{1}{2 \pi} \int_{-\infty}^{\infty} c_{66}\left(R_{1}\left(B_{0}+\epsilon B_{1}\right) e^{\alpha_{1}|k| \Delta}+R_{2}\left(C_{0}+\epsilon C_{1}\right) e^{\alpha_{2}|k| \Delta}\right) e^{-i k y} d k$

Since $|\in f(y)| \ll 1$ therefore let

$e^{t|k| \in f(y)} \approx 1 \pm|k| \in f(y)$

Applying the Boundary Conditions (51) and using equations (53)-(61), we obtain the following equations:

Case 1: Zero order terms:

$$
\begin{aligned}
-B_{0} P_{1}-C_{0} P_{2}-D_{0} P_{1}-G_{0} P_{2} & =0 \\
& B_{0}+C_{0}-D_{0}-G_{0}=0 \\
-B_{0} Q_{1}-C_{0} Q_{2}+D_{0} Q_{1}+G_{0} Q_{2} & =-F_{0} \\
-B_{0} R_{1}-C_{0} R_{1}-D_{0} R_{1}-G_{0} R_{2} & =0
\end{aligned}
$$

Therefore, the zero order solution is

$$
B_{0}=-C_{0}=-D_{0}=G_{0}=-\frac{F_{0}}{2\left(Q_{2}-Q_{1}\right)}
$$

Where

$$
Q_{2}-Q_{1}=d_{11}\left(P_{2} \alpha_{2}-P_{1} \alpha_{1}\right)|k|
$$

Case 2: First order terms:

The first order terms are evaluated as:

$$
\begin{aligned}
& B_{1}=-\frac{F_{0}}{2 \sigma_{11}\left(p_{2} \alpha_{2}-P_{1} \alpha_{1}\right)|k|}-\frac{F_{0} \alpha_{1} f(y)}{2 \sigma_{11}\left(P_{2} \alpha_{2}-P_{1} \alpha_{1}\right)} \\
& C_{1}=\frac{F_{0}}{2 \sigma_{11}\left(P_{2} \alpha_{2}-P_{1} \alpha_{1}\right)|k|}+\frac{F_{0} \alpha_{2} f(y)}{2 \sigma_{11}\left(p_{2} \alpha_{2}-P_{1} \alpha_{1}\right)} \\
& D_{1}=\frac{F_{0}}{\left.2 \sigma_{11}\left(P_{2} \alpha_{2}-P_{1} \alpha_{1}\right) \mid k\right]}+\frac{F_{0} \alpha_{1} f(y)}{2 \sigma_{11}\left(P_{2} \alpha_{2}-P_{1} \alpha_{1}\right)} \\
& G_{1}=-\frac{F_{0}}{2 \sigma_{11}\left(P_{2} \alpha_{2}-P_{1} \alpha_{1}\right)|k|}-\frac{F_{0} \alpha_{2} f(y)}{2 \sigma_{11}\left(P_{2} \alpha_{2}-P_{1} \alpha_{1}\right)}
\end{aligned}
$$

Using equations $(63)-(66)$ in (53) we obtain the values as follows:

$$
\begin{aligned}
& B=\frac{-F_{0}}{2 \sigma\left(P_{2} \alpha_{2}-P_{1} \alpha_{1}\right)|K|}-\in\left(\frac{F_{0}}{\left.2 \sigma_{11}\left(P_{2} \alpha_{2}-P_{1} \alpha_{1}\right) \mid k\right]}+\frac{F_{0} \alpha_{1} f(y)}{2 \sigma_{11}\left(P_{2} \alpha_{2}-P_{1} \alpha_{1}\right)}\right) \\
& C=\frac{F_{0}}{2 \sigma_{11}\left(P_{2} \alpha_{2}-P_{1} \alpha_{1}\right)|K|}+\in\left(\frac{F_{0}}{2 \sigma_{11}\left(P_{2} \alpha_{2}-P_{1} \alpha_{1}\right)|k|}+\frac{F_{0} \alpha_{2} f(y)}{2 \sigma_{11}\left(P_{2} \alpha_{2}-P_{1} \alpha_{1}\right)}\right) \\
& D=\frac{F_{0}}{2 \sigma_{11}\left(P_{2} \alpha_{2}-P_{1} \alpha_{1}\right)|K|}+\in\left(\frac{F_{0}}{2 c_{11}\left(P_{2} \alpha_{2}-P_{1} \alpha_{1}\right)|k|}+\frac{F_{0} \alpha_{1} f(y)}{2 c_{11}\left(P_{2} \alpha_{2}-P_{1} \alpha_{1}\right)}\right) \\
& G=\frac{-F_{0}}{2 \varepsilon_{11}\left(P_{2} \alpha_{2}-P_{1} \alpha_{1}\right)|K|}-\in\left(\frac{F_{0}}{2 \varepsilon_{11}\left(P_{2} \alpha_{2}-P_{1} \alpha_{1}\right)|k|}+\frac{F_{0} \alpha_{2} f(y)}{2 \varepsilon_{11}\left(P_{2} \alpha_{2}-P_{1} \alpha_{1}\right)}\right)
\end{aligned}
$$

\subsection{Rectangular Irregularity}

By applying Fourier transformation techniques on equation (1) we obtained

$$
\bar{f}(k)=\frac{4 a}{k} \sin (k a)
$$

Therefore by using inverse transformation we have:

$$
f(y)=\frac{1}{2 \pi} \int_{-\infty}^{\infty} \frac{4 a}{k} \sin (k a) e^{-i k y} d k=\operatorname{sign}(\mathrm{a}-\mathrm{y})+\operatorname{sign}(\mathrm{a}+\mathrm{y})
$$

where $\mathrm{E}=\frac{h}{2 a}$ and sign is the signum function.

By substituting the values of constants B, C, D, G from equation (67) in the equations (53) - (61) and value of $\mathrm{f}(\mathrm{y})$ for Rectangular irregularity from equation (68) we obtain the displacements and stresses in the integral form. After using the appendix we obtain the following closed form analytical expressions for the deformation field when the rectangular irregularity present in the orthotropic elastic medium: 
$u^{N}(x, y)=\frac{F_{0}}{4 \pi c_{11}\left(P_{2} \alpha_{2}-P_{1} \alpha_{1}\right)}\left[P_{1} \log S_{1}^{2}-P_{2} \log S_{2}^{2}+E\left\{P_{1} x \log S_{1}^{2}-\frac{P_{2}}{2 a} x \log S_{2}^{2} \mp \frac{x^{2}}{a}[\operatorname{sign}(a-y)+\right.\right.$ $\left.\left.\operatorname{sign}(a+y)]\left(\frac{P_{1} \alpha_{1}^{2}}{S_{1}^{n}}-\frac{P_{2} \alpha_{2}^{2}}{S_{2}^{n}}\right)\right\}\right]$

$v^{N}(x, y)=\mp \frac{F_{0}}{4 \pi c_{11}\left(P_{2} \alpha_{2}-P_{1} \alpha_{1}\right)}\left[\log S_{1}^{2}-\log S_{2}^{2}+E\left\{-\frac{x}{a} \log S_{1}^{2}+\frac{x}{a} \log S_{2}^{2} \pm x^{2}[\operatorname{sign}(a-y)+\right.\right.$ $\left.\left.\operatorname{sign}(a+y)]\left(\frac{\alpha_{1}^{2}}{S_{1}^{2}}-\frac{\alpha_{2}^{2}}{S_{2}^{2}}\right)\right\}\right]$

$\sigma_{x}^{N}(x, y)=\frac{F_{0}}{4 \pi c_{11}\left(P_{2} \alpha_{2}-P_{1} \alpha_{1}\right)}\left[ \pm \frac{2 P_{1} \alpha_{1}^{2} d_{11} x}{S_{1}^{n}}-\frac{2 d_{12} y}{S_{1}^{n}} \mp \frac{2 d_{11} \alpha_{2}^{2} x P_{2}}{S_{2}^{2}}+\frac{2 d_{12} y}{S_{2}^{2}}+E\left\{\frac{ \pm \alpha_{1}^{2} d_{11} x^{2}}{S_{1}^{2}}-\frac{d_{12} x y}{S_{1}^{n}} \mp\right.\right.$

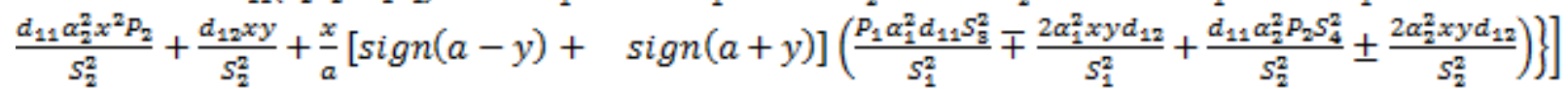

$\tau_{x y}^{N}(x, y)=\frac{F_{0}}{4 \pi c_{11}\left(P_{2} \alpha_{2}-P_{1} \alpha_{1}\right)}\left[\mp \frac{2 \alpha_{1}^{2} x}{S_{1}^{2}}+\frac{2 P_{1} y}{S_{1}^{2}} \pm \frac{2 \alpha_{2}^{2} x}{S_{2}^{2}}-\frac{2 P_{2} y}{S_{2}^{n}}+E\left\{\mp \frac{\alpha_{1}^{2} x^{2}}{a S_{1}^{n}}+\frac{P_{1} x y}{a S_{1}^{n}} \pm \frac{\alpha_{2}^{2} x^{2}}{a S_{2}^{2}}-\frac{p_{2} x y}{a S_{2}^{2}}+\right.\right.$ $\left.\left.\frac{x}{a}[\operatorname{sign}(a-y)+\operatorname{sign}(a+y)]\left(\frac{-\alpha_{1}^{n} S_{a}^{n}}{S_{1}^{n}} \mp \frac{2 \alpha_{1}^{n} x y P_{1}}{S_{1}^{n}}+\frac{\alpha_{2}^{n} S_{4}^{n}}{S_{2}^{n}} \pm \frac{2 \alpha_{2}^{n} x y P_{2}}{S_{2}^{n}}\right)\right\}\right]$

\subsection{Parabolic Irregularity}

Here by using equation (2) and (18), we obtain

$\bar{f}(k)=4 a\left(\frac{\sin (k a)-a k \cos (k a)}{k^{3} a^{s}}\right)$

therefore by using inverse transformation we have

$f(y)=\frac{1}{2 \pi} \int_{-\infty}^{\infty} 4 a\left(\frac{\sin (k a)-a k \cos (k a)}{k^{\mathrm{s}} a^{\mathrm{s}}}\right) e^{-i k y} d k=\left(1-\frac{y^{2}}{a^{2}}\right)[\operatorname{sign}(a-y)+\operatorname{sign}(a+y)]$

$\& \in f(y)=h\left(1-\frac{y^{2}}{a^{2}}\right), \in=\frac{h}{2 a}$

Similarly as above, we obtain the following closed form analytical expressions for the deformation field when the parabolic irregularity present in the orthotropic elastic medium:

$u^{N}(x, y)=\frac{F_{0}}{4 \pi c_{11}\left(P_{2} \alpha_{2}-P_{1} \alpha_{1}\right)}\left[P_{1} \log S_{1}^{2}-P_{2} \log S_{2}^{2}+E\left\{\frac{P_{1}}{a} x \log S_{1}^{2}-\frac{P_{2}}{a} x \log S_{2}^{2} \mp x^{2}(1-\right.\right.$

$\left.\left.\left.\frac{y^{2}}{a^{2}}\right)[\operatorname{sign}(a-y)+\operatorname{sign}(a+y)]\left(\frac{P_{1} \alpha_{1}^{2}}{S_{1}^{2}}-\frac{P_{2} \alpha_{2}^{2}}{S_{2}^{2}}\right)\right\}\right]$

$v^{N}(x, y)=\mp \frac{F_{0}}{4 \pi c_{11}\left(P_{2} \alpha_{2}-P_{1} \alpha_{1}\right)}\left[\log S_{1}^{2}-\log S_{2}^{2}+E\left\{-\frac{x}{a} \log S_{1}^{2}+\frac{x}{a} \log S_{2}^{2} \pm x^{2}\left(1-\frac{y^{2}}{a^{2}}\right)[\operatorname{sign}(a-\right.\right.$ $\left.\left.y)+\operatorname{sign}(a+y)]\left(\frac{\alpha_{1}^{2}}{s_{1}^{2}}-\frac{\alpha_{2}^{2}}{S_{1}^{2}}\right)\right\}\right]$

$\sigma_{x}^{N}(x, y)=\frac{F_{0}}{4 \pi c_{11}\left(P_{2} \alpha_{2}-P_{1} \alpha_{1}\right)}\left[ \pm \frac{2 P_{1} \alpha_{1}^{2} d_{11} x}{S_{1}^{n}}-\frac{2 d_{12} y}{s_{1}^{n}} \mp \frac{2 d_{11} \alpha_{2}^{2} x P_{2}}{S_{2}^{n}}+E\left\{\frac{ \pm \alpha_{1}^{2} d_{11} x^{2}}{S_{1}^{n}}-\frac{d_{12} x y}{S_{1}^{n}} \mp \frac{d_{11} \alpha_{2}^{2} x^{2} P_{2}}{S_{2}^{n}}+\right.\right.$ $\left.\left.\frac{d_{12} x y}{S_{2}^{n}}+\left[\operatorname{sign}(a-y)+x\left(1-\frac{y^{2}}{a^{2}}\right) \operatorname{sign}(a+y)\right]\left(\frac{P_{1} \alpha_{1}^{2} d_{11} S_{2}^{n}}{s_{1}^{2}} \mp \frac{2 \alpha_{1}^{n} x y d_{12}}{s_{1}^{n}}+\frac{d_{11} \alpha_{2}^{2} p_{2}^{2} S_{4}^{n}}{s_{2}^{n}} \pm \frac{2 \alpha_{2}^{2} x y d_{12}}{s_{2}^{n}}\right)\right\}\right]$

$\tau_{x y}^{N}(x, y)=\frac{F_{0}}{4 \pi c_{11}\left(P_{2} \alpha_{2}-P_{1} \alpha_{1}\right)}\left[\mp \frac{2 \alpha_{1}^{2} x}{S_{1}^{n}}+\frac{2 P_{1} y}{S_{1}^{x}} \pm \frac{2 \alpha_{2}^{2} x}{S_{2}^{n}}-\frac{2 P_{2} y}{S_{2}^{n}}+E\left\{\mp \frac{\alpha_{1}^{2} x^{2}}{a S_{1}^{n}}+\frac{P_{1} x y}{a S_{1}^{2}} \pm \frac{\alpha_{2}^{2} x^{2}}{a S_{2}^{2}}-\frac{p_{2} x y}{a S_{2}^{2}}+\right.\right.$ $\left.\left.x\left(1-\frac{y^{2}}{a^{2}}\right)[\operatorname{sign}(a-y)+\operatorname{sign}(a+y)]\left(\frac{-\alpha_{1}^{2} R_{1}^{2}}{s_{1}^{n}} \mp \frac{2 \alpha_{1}^{2} x y P_{1}}{s_{1}^{2}}+\frac{\alpha_{2}^{2} R_{4}^{2}}{S_{2}^{2}} \pm \frac{2 \alpha_{2}^{2} x y P_{2}}{s_{2}^{n}}\right)\right\}\right]$

where $\mathrm{E}=\frac{h}{x}$ is the parameter of irregularity and 
$S_{1}^{2}=y^{2}+\alpha_{1}^{2} x^{2}, S_{2}^{2}=y^{2}+\alpha_{2}^{2} x^{2}, S_{3}^{2}=\alpha_{1}^{2} x^{2}-y^{2}, S_{4}^{2}=\alpha_{2}^{2} x^{2}-y^{2}$

On taking $\mathrm{E}=0$ (in the absence of irregularity ) the corresponding results for regular orthotropic elastic medium can be obtained from the above results as a particular case which coincide with the results obtained by Garg et al. [8].

\section{Numerical Results and Discussion}

We intend to examine the effect of irregularities due to normal line loading in an orthotropic elastic medium. The irregularity is either Rectangular or Parabolic at the interface of an elastic medium. For numerical computation we use the values of elastic constants given by Verma [15] for Olivin material which are
$d_{11}=324.0$
$d_{22}=198.0$,
$d_{33}=249.0$,
$d_{12}=59.0$
$d_{23}=78.0$,
$d_{31}=79.0$,
$d_{66}=79.3$,
$d_{44}=66.7$,
$d_{55}=81.0$

in terms of unit stress of $10^{11}$ dynes $\mathrm{cm}^{-2}$.

The effect of irregularities on the displacement due to normal line-load acting vertically downwards on the irregular interface of orthotropic elastic medium are plotted in Figures (2) \& (3). The irregularities consider is either rectangular or parabolic. Figures (2) \& (3) corresponds to the variation of normal displacement with the horizontal distance for different sizes of rectangular and parabolic irregularity respectively, $(\mathrm{E}=0.2,0.4$ and 0.6$)$ and of regular shape (in the absence of irregularity) of the medium i.e. $\mathrm{E}=0$.

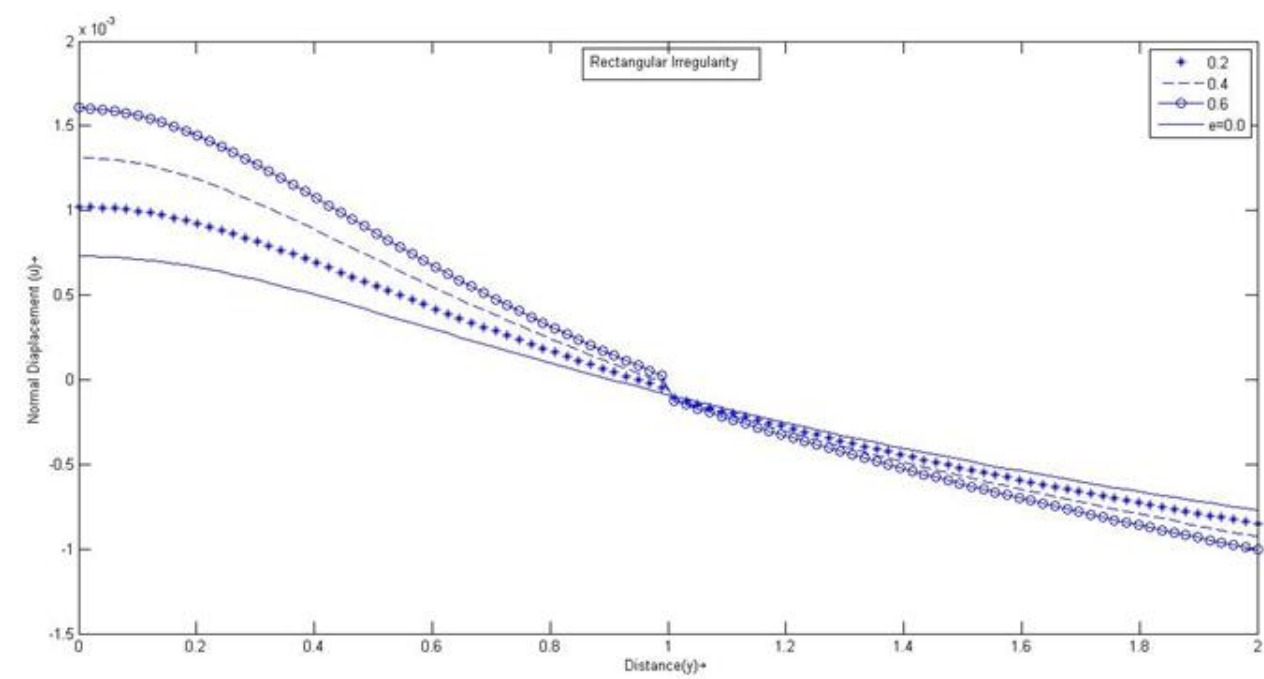

Fig. 2. Variation of normal displacement $(u)$ with the horizontal distance $(y)$ on the plane at $x=1.0$ at size of $\mathrm{E}=0.2,0.4$ and 0.6 of rectangular irregularity.

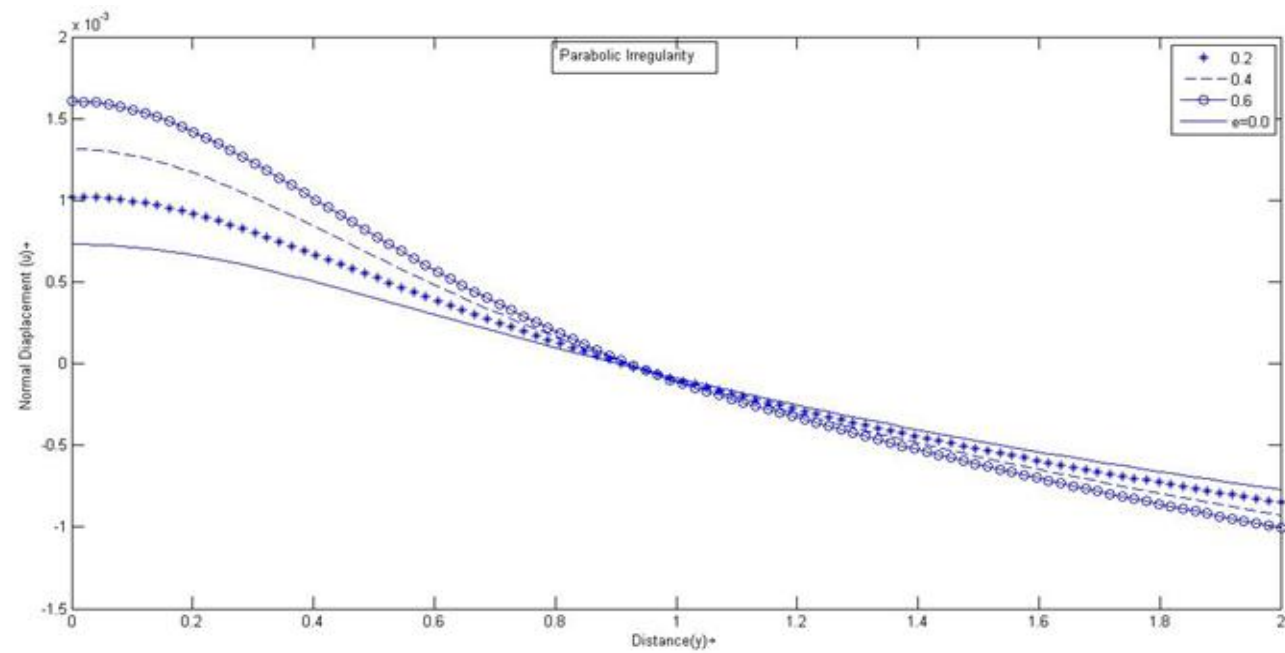

Fig. 3. Variation of normal displacement $(u)$ with the horizontal distance $(y)$ on the plane at $x=1.0$ at size of $\mathrm{E}=0.2,0.4$ and 0.6 of parabolic irregularity. 
For the irregular size $\mathrm{E}=0.2,0.4,0.6$, the comparison between the displacements due to presence of rectangular and parabolic irregularities in orthotropic elastic medium have been shown in figure (4)-(6). From these figures it has been observed that the displacements due to rectangular and parabolic irregularities are equal at $\mathrm{y}=0$ and the difference increases till the end of the irregularity. Also in magnitude the displacement due to rectangular irregularity is greater than that of the due to parabolic irregularity present in orthotropic elastic medium.

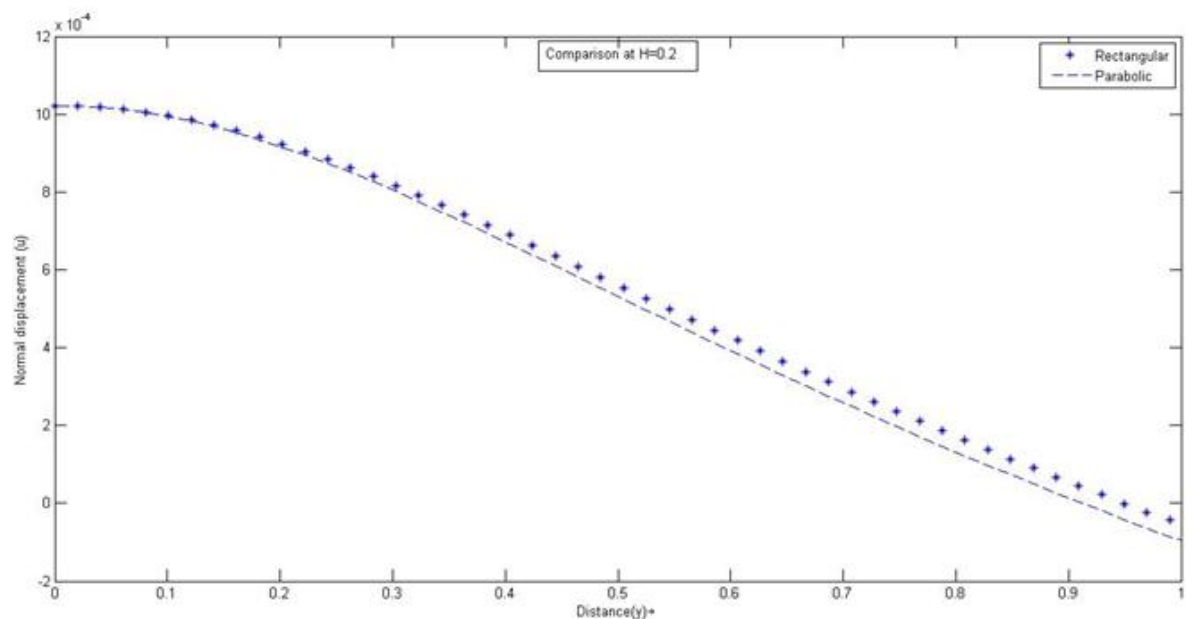

Fig. 4. Variation of normal displacement $(u)$ with the horizontal distance $(y)$ for rectangular and parabolic irregularities present in orthotropic elasic medium at $\mathrm{E}=0.2$

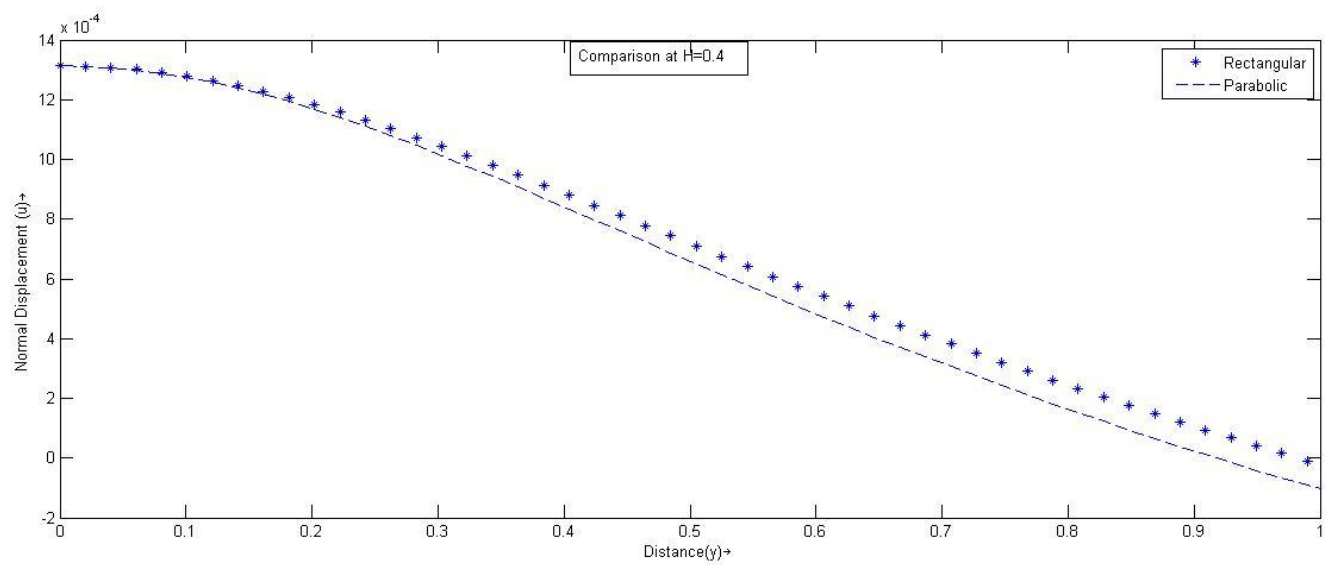

Fig. 5. Variation of normal displacement $(u)$ with the horizontal distance $(y)$ for rectangular and parabolic irregularities present in orthotropic elastic medium at $\mathrm{E}=0.4$

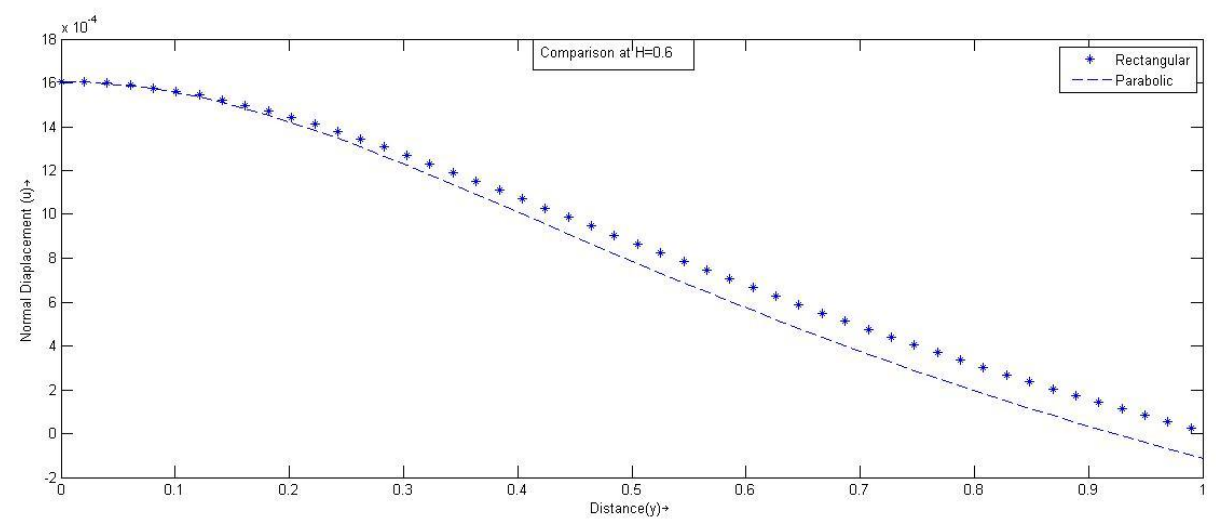

Fig. 6. Variation of normal displacement $(u)$ with the horizontal distance $(y)$ for rectangular and parabolic irregularities present in orthotropic elastic medium at $\mathrm{E}=0.6$ 
Contour maps for the normal displacement due to rectangular and parabolic irregularities with $\mathrm{E}=0.2$, 0.4, 0.6 (sizes of irregularities) on the distance depth grid are depicted in Figures (7) \& (8). Figures (9) \& (10), correspond to the variation of normal stresses with the horizontal distance y for rectangular and parabolic irregularities at $\mathrm{E}=0.2,0.4,0.6$. From these figures frequent intercrossing of various curves for different values of $\mathrm{E}$ are shown.

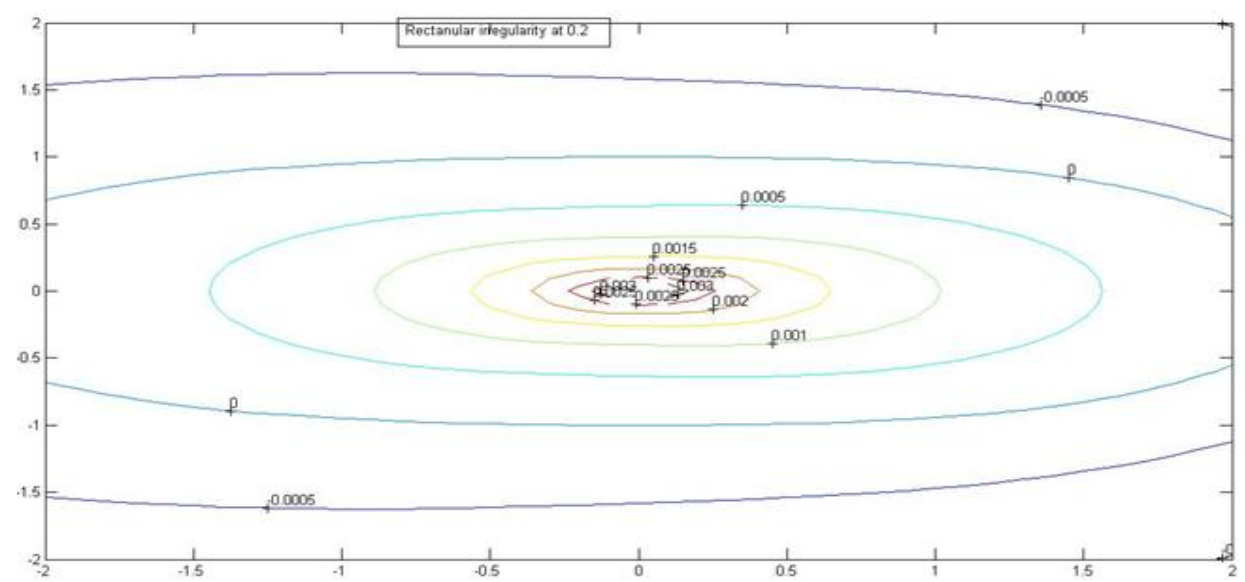

Fig. 7(a)

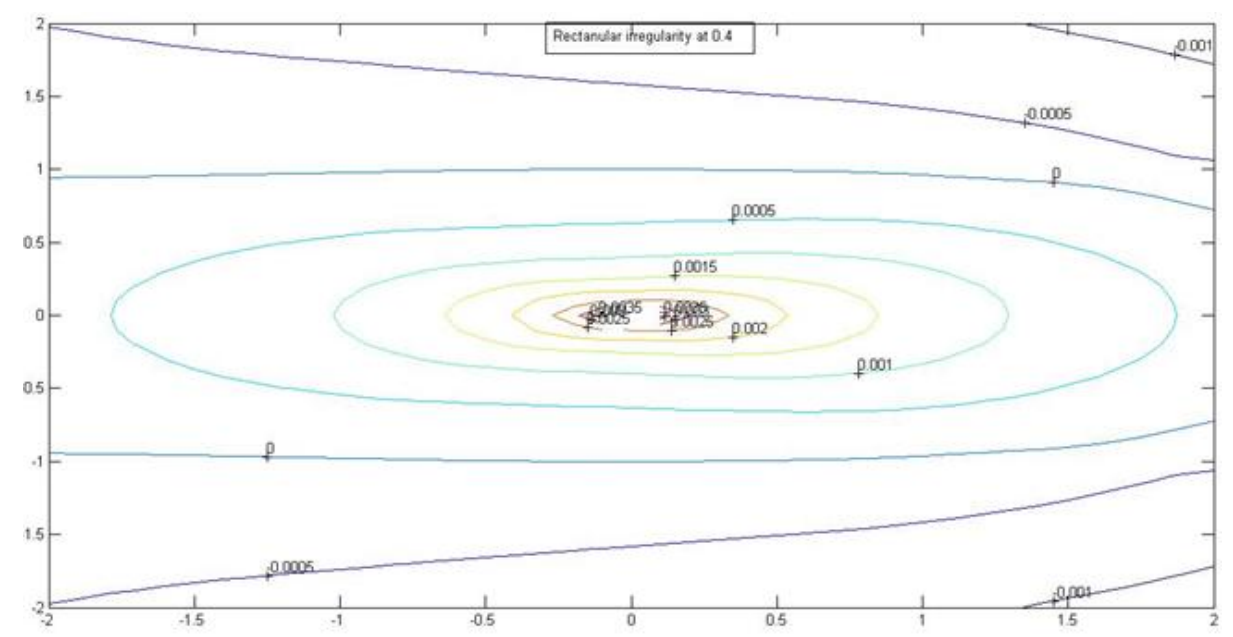

Fig. 7(b)

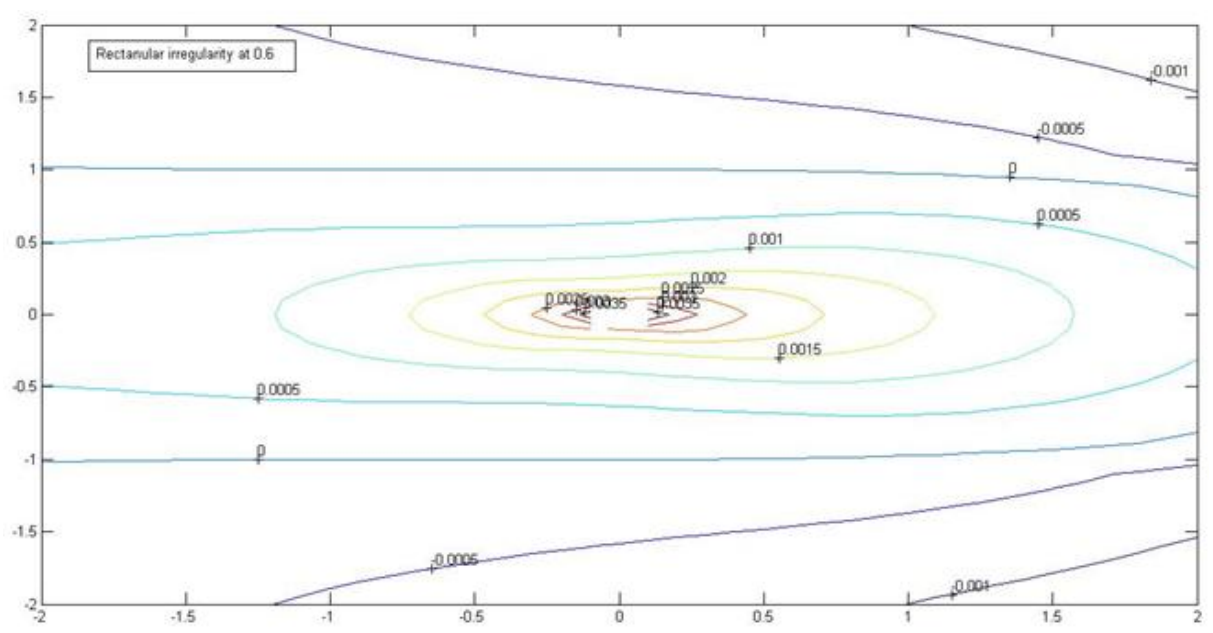

Fig. 7 (c)

Fig. 7. Contour Map for the displacement with rectangular irregularity for (a) $E=0.2$, (b) $E=0.4$ ), (c) $E=0.6$ 


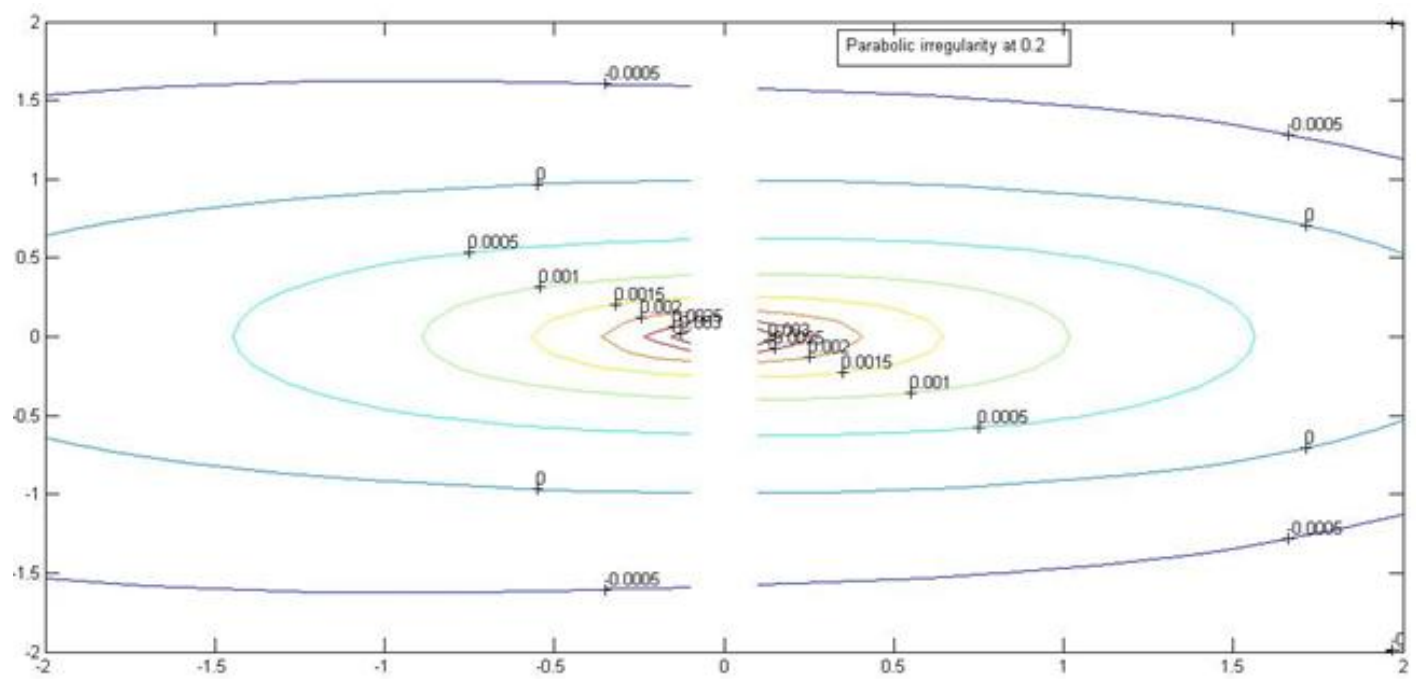

Fig. 8(a)

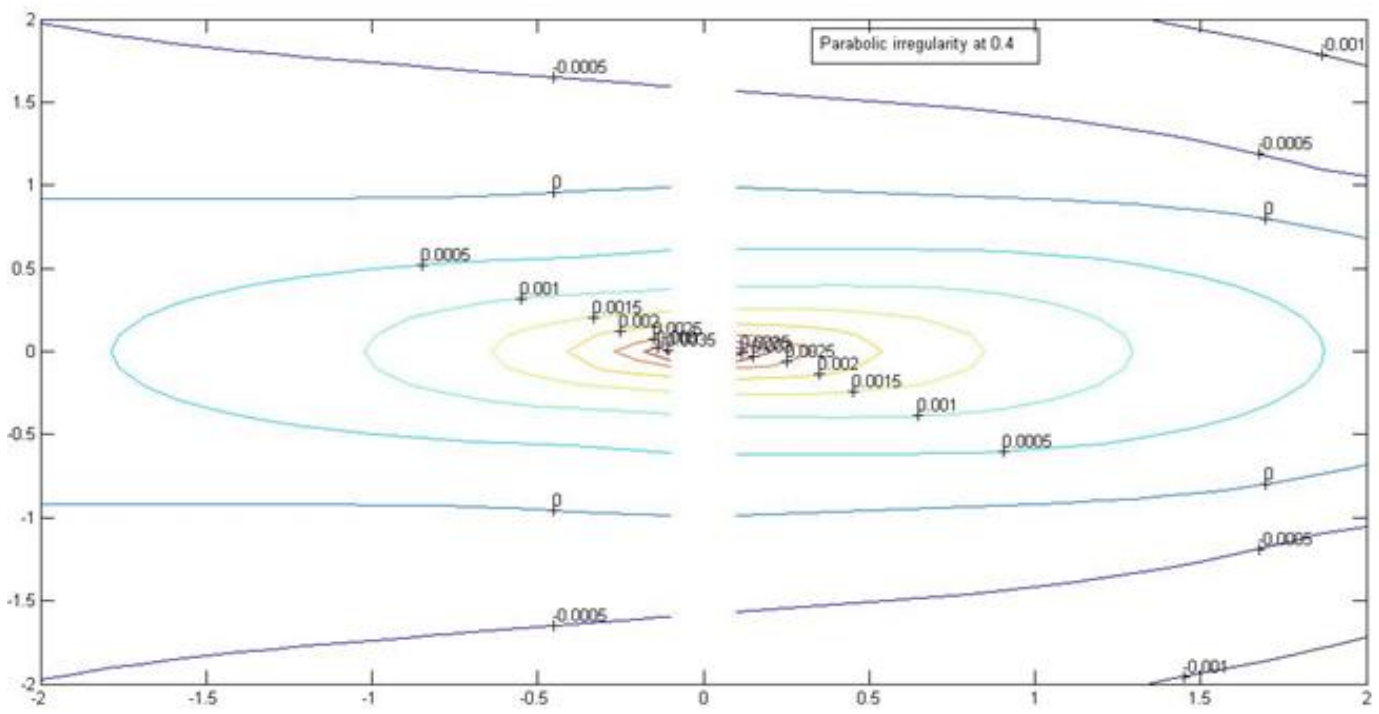

Fig. 8(b)

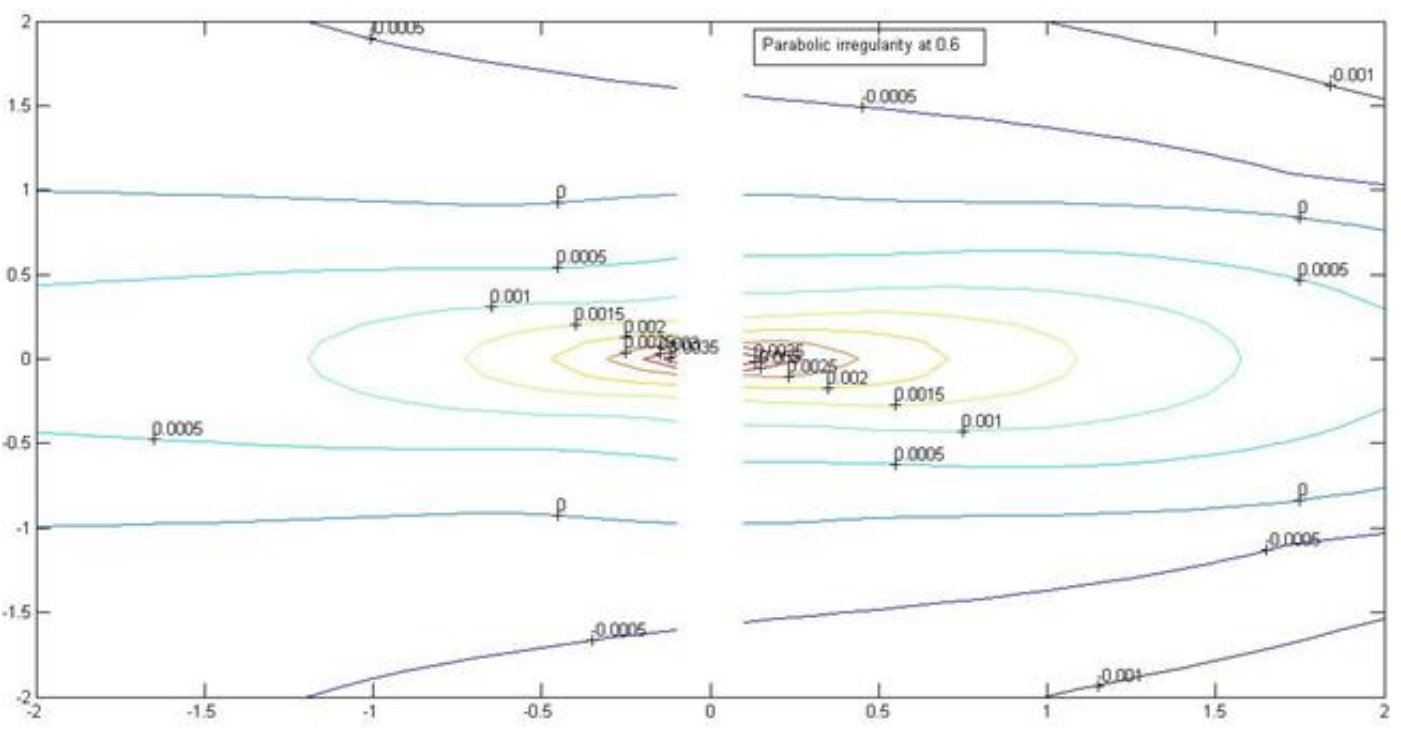

Fig. 8(c)

Fig. 8. Contour Map for the displacement with parabolic irregularity for (a) $E=0.2$, (b) $E=0.4$ ), (c) $E=0.6$ 


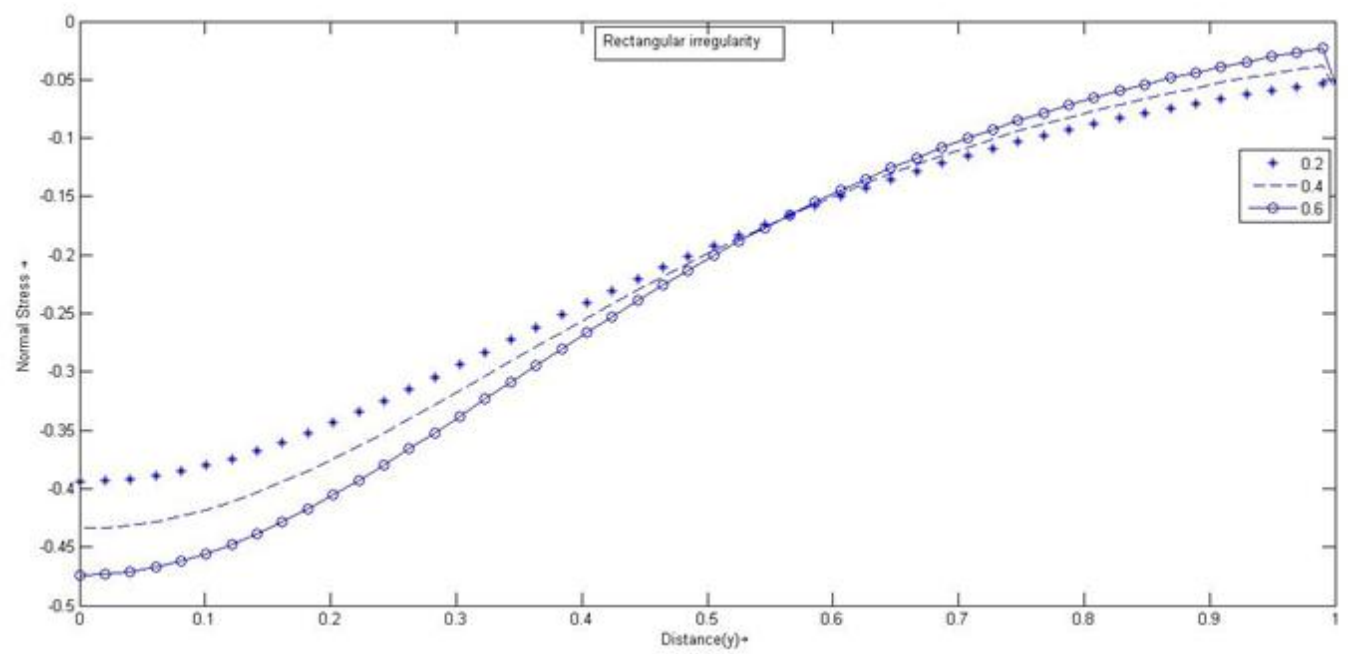

Fig. 9. Variation of normal stress with the horizontal distance $(y)$ on the plane at $x=1.0$ at size of $E=0.2,0.4$ and 0.6 of rectangular irregularity.

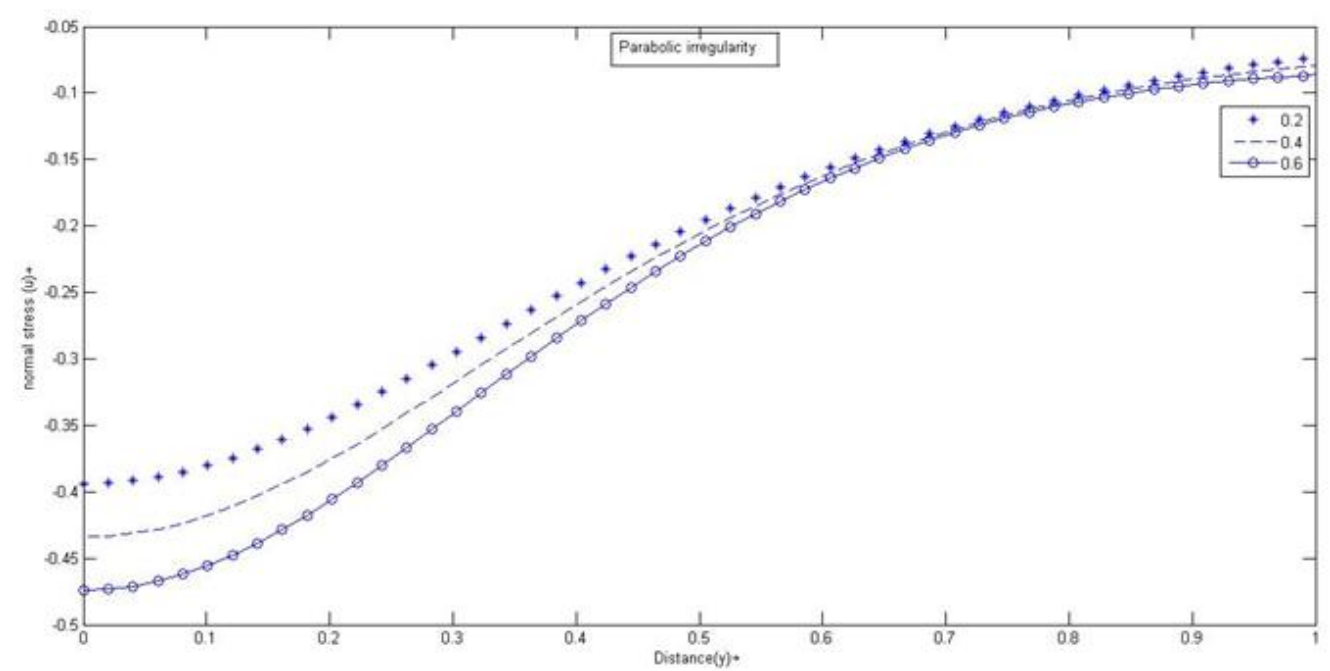

Fig. 10. Variation of normal stress with the horizontal distance $(y)$ on the plane at $x=1.0$ at size of $E=0.2,0.4$ and 0.6 of parabolic irregularity.

$$
\begin{aligned}
& \int_{-\infty}^{\infty} \exp (-|k| x) \exp (-i k y) d k=\frac{2 x}{y^{2}+x^{2}} \\
& \int_{-\infty}^{\infty} \frac{k}{|k|} \exp (-|k| x) \exp (-i k y) d k=\frac{-2 i y}{y^{2}+x^{2}} \\
& \int_{-\infty}^{\infty}(|k|)^{-1} \exp (-|k| x) \exp (-i k y) d k=-\log \left(y^{2}+x^{2}\right) \\
& \int_{-\infty}^{\infty}|k| \exp (-|k| x) \exp (-i k y) d k=\frac{2\left(x^{2}-y^{2}\right)}{\left(y^{2}+x^{2}\right)^{2}} \\
& \int_{-\infty}^{\infty} k \exp (-|k| x) \exp (-i k y) d k=\frac{-4 i y x}{\left(y^{2}+x^{2}\right)^{2}} \\
& \int_{-\infty}^{\infty} \frac{1}{k} \exp (-|k| x) \exp (-i k y) d k=-2 i t a n^{-1}\left(\frac{y}{x}\right)
\end{aligned}
$$

\section{Acknowledgement}

One of the authors(DKM) is thankful to University Grant Commission, New Delhi for Major Research Project vide F.No.43-437/2014 (SR). 


\section{References}

[1]. J. D. Noyer, The effect of variations in layer thickness of love waves, Bull. Seism. Soc. Am.51, 1961 $227-235$.

[2]. A.K Mal, On the frequency equation for love waves due to abrupt thickening of crustal layer, Geofis .Pure Appl.52, 59, 1962, 5968.

[3]. B.K.Kar ,A.K. Pal and V.K. Kalyani ,Propagation of love waves in an isotropic dry sandy layer ,Acta Geophys 34 (2) ,157, 1986, $157-170$.

[4]. A.Chattopadhyay,.Chakraborty and A.K. Pal, Effect of irregularity on the propagation of guided SH-waves, J. Mecan, Theor. Appl (France) 2(2), 1983, 215-225.

[5]. D P Acharya and Indrajit Roy, Effect of surface stress and irregularity of the interface on the propagation of SH-waves in the magneto- elastic crustal layer based on a solid semi space; Sadhana 34 (2), 2009, 309-330.

[6]. D. K. Madan , R. Kumar, J.S. Sikka ,Love wave propagation in an irregular fluid saturated porous anisotropic layer with rigid boundaries, Journal of Applied Science and Research 10(4), 2014 281-287.

[7]. R. Kumar, D. K .Madan, J.S Sikka , Shear wave propagation in multilayered medium including an irregular fluid saturated porous stratum with rigid boundary, Advances in Mathematical Physics, http://dx.doi.org/10.1155/2014/163505 , 2014, 1-9.

[8]. N.R. Garg, R. Kumar,A. Goel and A. Miglani, Plain strain deformation of an orthotropic elastic medium using an eigen value approach, Earth Planets Space,55 (3) , 2003, 3-9.

[9]. K.Singh,D.K.Madan ,A.Goel and N.R.Garg ,Two-dimensional static deformation of anisotropic medium ,Sadhana 30(4), 565 , 2005, 565-583.

[10]. M.M.Selim, Effect of irregulatiry on static deformation of elastic half-space, International Journal of Modern Physics ,22(14) , $2008,2241-2253$.

[11]. A.K.Mal and S.J. Singh, Deformation of elastic solids,( Prentice Hall, 1991).

[12]. L.Debnath, Integrat Transforms and their Applications, (CRC Press. Inc.,New York ,1995).

[13]. R.G.Payton, Elastic wave propagation in transversely isotropic media, Martinus Nijhoff Publishers. The Hague, 1983.

[14]. A.C. Eringen and E.S.Suhubi ,Elastodynamics $x$ vol II (Academic Press ,New York, 1975).

[15]. R.K Verma , Elasticity of some high density crystals , Journal of Geological Research, 65 (2), 1960, 757-766.. 